\title{
Thank You to Our Reviewers
}

We are indebted to the expert referees who have volunteered their time to review submissions for The Canadian Journal of Neurological Sciences in 2018. The editors, authors and readers are immensely grateful for their thoughtfulness and expertise that have served our journal well.

\author{
Abulhasan, Yasser \\ Al-Hertani, Walla \\ Alikhani, Katayoun \\ Allen, J \\ Altman, Robert \\ Andrade, Danielle \\ Andrews, Saadet \\ Angel, Michael \\ Appel-Cresswell, Silke \\ Au, Karolyn \\ Ba, Fang \\ Bajunaid, Khalid \\ Baker, Steven \\ Bayley, Mark \\ Becker, Werner \\ Berciano, J \\ Berlit, Peter \\ Bhargava, Ravi \\ Bhaskar, Sonu \\ Biller, Jose \\ Blacquiere, Dylan \\ Boelman, Cyrus \\ Boex, Colette \\ Boulos, Mark \\ Boulton, Mel \\ Bourque, Pierre \\ Boyd, John \\ Breen, David \\ Breiner, Ari \\ Bronchti, Gilles \\ Brouillette, Jonathan \\ Brown, Matthew \\ Bruno, Veronica \\ Brust, Tyson \\ Buchsbaum, Brad \\ Buck, Brian \\ Budhram, Adrian \\ Bunce, Paul \\ Burke, Tom \\ Burneo, Jorge \\ Burton, Jodie \\ Camfield, Peter \\ Camicioli, Richard \\ Carey, Robert \\ Carruthers, Robert \\ Carter, Melissa \\ Chalil, Alan \\ Chalk, Colin \\ Chan, David \\ Chan, Ming
}

\author{
Chan, Vivien \\ Chen, Joyce \\ Chen, Kai-Hsiang Stanley \\ Chen, Kevin \\ Choi, Victor \\ Chow, Michael \\ Chum, Marvin \\ Climans, Seth \\ Cloutier, Martin \\ Connolly, Barbara \\ Connolly, Mary \\ Cooke, Lara \\ Cooper, Paul \\ Cortes, Maria \\ Costello, Fiona \\ Culpepper, William \\ Dakson, Ayoub \\ Dancause, Numa \\ Dardis, C \\ Das, Sumit \\ Datta, Anita \\ Davis, Karen \\ Day, Gregory \\ Dea, Nicolas \\ Debert, Chantel \\ Debicki, Derek \\ Deis, Nathan \\ Delport, Johan \\ Di Maio, Salvatore \\ Diringer, Michael \\ Dromey, Christopher \\ Ducharme, Simon \\ Dukelow, Sean \\ Dunham, Christopher \\ Duquette, Pierre \\ Eagles, Matthew \\ Ehrensperger, Eric \\ Eisenstat, David \\ Elliott, Cameron \\ Fang-Cheng, Yeh \\ Fasano, Alfonso \\ Federico, Paolo \\ Fehlings, Darcy \\ Ferro, JM \\ Fiest, Kirsten \\ Finger, Elizabeth \\ Finsterer, Josef \\ Florendo-Cumbermack, \\ Anita \\ Fox, Richard
}

França, Marcondes Jr

Freedman, Mark

Fridman, Sebastian

Furlan, Julio

Gasca-Salas, Carmen

Gaspard, Nicolas

Gazulla, José

Genge, Angela

Gladstone, Jonathan

Go, Tina

Gofton, Teneille

Gonarazky, Hernan

Gorassini, Monica

Gotman, Jean

Goulet, Kristian

Green, Robin

Green, Theresa

Grimes, David

Gris, Denis

Grisold, W

Guo, Meiqi

Habek, Mario

Hahn, Cecil

Hahn, Christopher

Hammond, R

Hazrati, Lili-Naz

Henri-Bhargava, Alexandre

Hodaie, Mojgan

Honey, Christopher

Horvath, Gabriella

Hsiung, Robin

Hubertus, Axer

Hussain, Mohammed

Hyland, Keith

Inbar-Feigenberg, Michal

Iorio-Morin, Christian

Ismail, Zahinoor

Izenberg, Aaron

Jackson, Alan

Jacobs, W Bradley

Jichici, Draga

Jones, Kevin

Joseph, Jeffrey

Josephson, Colin

Joshi, Sucheta

Kaderali, Zul

Kalia, Suneil

Kasarskis, E

Kassubek, Jan

Katzberg, Hans
Kazi, Julhash

Kazina, Colin

Keezer, Mark

Kelly, Michael

Kesarwani, Rohit

Khaw, Alexander

Kirk, Andrew

Kirschner, J

Ko, Ji Hyan

Ko, Sang-Bae

Kondziella, Daniel

Korngut, Lawrence

Krings, Timo

Krishnan, Pradeep

Kulkarni, Abhaya

Kumar, Hrishikesh

Kumar, Sanjeev

Kutsogiannis, Demetrios

Kwan, Benjamin

Lafontaine, Anne-Louise

Lanthier, Sylvain

Lay, Christine

Lee, Donald

Lee, Liesly

Levy, Ariel

Li, Y

Lidstone, Sarah

Lipsman, Nir

Lizarraga, Karlo

Lo, Alexander

Lo, Yew Long

Lownie, Stephen

Lynch, Peter

MacGregor, Daune

Mahmoud, Sherif

Malhotra, Konark

Mandzia, Jennifer

Mao-Draayer, Yang

Marcoux, Judith

Marion, Travis

Marras, Connie

Martin, Wayne

Mason, Warren

Massie, Rami

Mathieu, David

Matte, Genevieve

Maurice, Catherine

Mayer, Stephan

McDonald, Patrick

McLachland, Richard 


\author{
McNamara, Mairead \\ McNeil, Stephen \\ Medhi, Gorky \\ Megyesi, Joseph \\ Meltzer, Jed \\ Mestre, Tiago \\ Metz, Luanne \\ Mikulis, David \\ Miller, Tom \\ Minami, Tetsuto \\ Minuk, Jeffrey \\ Mirsatarri, Seyed \\ Mitha, Alim \\ Mittal, Nimish \\ Mohammad, Shekeeb \\ Mohammed, Safraz \\ Morgante, Francesca \\ Morrow, Sarah \\ Munhoz, Renato \\ Munoz, David \\ Nagarajan, Lakshmi \\ Nair, Jay Kumar \\ Nascimento, Fabio \\ Nataraj, Andrew \\ $\mathrm{Ng}$, Marcus \\ Nguyen, Dang \\ Nicolle, Michael \\ Nijjar, Satnam \\ Noppens, Ruediger \\ OKelly, Cian \\ $\mathrm{Oh}, \mathrm{SH}$ \\ Ohorodnyk, Pavlo \\ Orr, Serena \\ Ortiz de la Rosa, JS \\ Pan, Edward \\ Para, Andrea \\ Parks, Natalie \\ Pelikan, Jonathan
}

\author{
Perez-Gonzalez, David \\ Perry, James \\ Pfeffer, Gerald \\ Phillips, Stephen \\ Phukan, Pranjal \\ Pickett, Gwynedd \\ Pikula, Aleksandra \\ Pillay, Neelan \\ Pohl, Daniella \\ Postuma, Ron \\ Power, Christopher \\ Prasad, Chitra \\ Pringle, Catherine \\ Purdy, Allan \\ Rafay, Mubeen \\ Rajput, Alex \\ Ramasubbu, Rajamannar \\ Rashiq, Saifee \\ Rastin, Tara \\ Reddy, Kesh \\ Redekop, Gary \\ Reis, Fabiano \\ Richardson, Denyse \\ Robinson, Larry \\ Rohani, Mohammad \\ Roldan Urgoiti, G \\ Rouleau, Guy \\ Rowe, Brian \\ Roy, Francois \\ Rubio, ME \\ Ryan, Robert \\ Ryu, Won Hyung \\ Sadler, Mark \\ Sahjpaul, Ramesh \\ Sakamuri, Sarada \\ Salehi, Fateme \\ Saluja, Rajeet \\ Sarnthein, Johannes
}

Sauro, Khara

Sayeed, Wajid

Schellenberg, Kerri

Schneider, Raphael

Schwartz, Christopher

Shankar, Jai

Sheikh, Shehnaz

Shevell, Michael

Shoesmith, Christen

Shulman, Richard

Siddiqi, Zaeem

Silverberg, Noah

Simpson, Robert

Slavin, K

Sleigh, J

Slow, Elizabeth

Soh, Derrick

Solomon, Gary

Sondheimer, Neal

Sposato, Luciano

Steinbok, Paul

Steriade, Claude

Steve, Trevor

Stewart, John

Stotts, Grant

Sundaram, Arun

Suppa, Antonio

Taranath, Ajay

Tang-Wai, David

Tarnutzer, Alexander

Tartaglia, Maria Carmela

Teasell, Robert

Tein, Ingrid

Tellez-Zenteno, JF

Terranova, Carmen

Thompson, Spencer

Topolovec-Vranic, Jane

Traboulsee, Anthony
Turnbull, John

Van Alfen, Nens

Vassilyadi, Michael

Venance, Shannon

Venkat

Raghavan, Lakshmikumar

Venkateswaran, Sunita

Vodopivec, I

Vázquez, Juan

Wagle Shukla, Aparna

Wang, David

Watson, C Peter

Wennberg, Richard

Willer, Barry

Wilson, Jefferson

Wong, John

Woulfe, John

Yeates, Keith

Yip, Stephen

Young, G Bryan

$\mathrm{Yu}$, Amy

Zeiler, Frederick

Zochodne, Douglas

Zurowski, Mateusz

Zwicker, Jocelyn

de Boysson, $\mathrm{H}$

de Guise, Elaine

deVries, Linda

del Campo, Martin

Grippo, Antonello

Lagman-Bartolome,

Ana-Marissa

Ramaswamy, Vijay

van Gerpen, Jay

Whitney, Robyn 